\title{
VERZEICHNIS DER SUCHPUNKTE
}

Die Nummern entsprechen denen am Rande des Textes sowie denen auf der Ùbersichtskarte

A $\quad 1$ Unstrut . . . . . . . 41

1.1 Der Fluß . . . . . . . 41

1.2 Die Wasserstraße . . . 47

2 Unstrutniederung . . . 49

3 Donndorf mit Kloster

Donndorf . . . . 55

4 Wiehe mit Hechendorf . 56

5 Wohlmirstedt mit Aller-

stedt. . . . . . . . . 61

6 Memleben . . . . . 63

7 Wendelstein . . . . . 68

8 Roßleben. . . . . . . 74

9 Hohe-Schrecke-Berg-

land . . . . . . . . 80

10 Kleinroda . . . . . . 81

11 Langenroda. . . . . . 82

12 Garnbach und Burgruine

Rabenswald . . . . . 82

13 Zeisdorf . . . . . . . 83

14 Schmücke-Rücken. . . 84

15 Lossa-Vippach-Hügel-

land . . . . . . 85

16 Finne . . . . . . . . 85

17 Lossa und Billroda . . 86

18 Rothenberga . . . . . 88

B 1 Bucha . . . . . . . . 88

2 Orlasberg und alte

Weinstraße. . . . . 90

3 Dissautal. . . . . . . 91

4 Altenroda mit Wippach und Birkigt. . . . . . 91

5 Saubach und Kahlwinkel mit Bernsdorf . 93

6 Bad Bibra . . . . . . 95

7 Bibertal . . . . . . . 98

8 Thalwinkel mit Bergwinkel und Golzen. . . 99

9 Bibraer Plateau. . . . 101
B 10 Naturschutzgebiet Forst

Bibra . . . . . . . . 101

11 Hirschroda, Plößnitz

und Krawinkel . . . . 104

12 Borntal . . . . . . 106

13 Wangener Unstruttal . 107

14 Naturschutzgebiet

Steinklöbe . . . . . . 108

15 Wangen . . . . . 110

16 Vogelherd . . . . . 113

17 Ziegelrodaer Plateau und Forst . . . . . . 113

18 Nebra-Lauchaer Unstruttal . . . . . 115

19 Nebra . . . . . . . . 118

20 Steilhänge bei Vitzenburg. . . . . . . . . 124

21 Reinsdorf und Zingst . 125

22 Karsdorf mit Wetzen-

dorf . . . . . . . . . 127

23 Wennungen. . . . . 132

24 Burgscheidungen . . . 133

25 Tröbsdorf . . . . . . 137

26 Kirchscheidungen . . . 139

C 1 Laucha mit Dorndorf . 141

2 Weischütz . . . . . . 147

3 Karsdorf-Dorndorfer

Stufenhang. . . . . 148

4 Trockenrasenhänge bei

Dorndorf .. . . . . . 149

5 Trockenrasenhänge bei

Karsdorf . . . . . . . 150

6 Müchelner und Querfurt-

Gleinaer Plateau . . . 151

7 Albersroda mit Schnell-

roda. . . . . . . . . 153

8 Baumersroda und

Ebersroda . . . . . . 154 
C 9 Gleina mit Müncheroda 155

10 Branderoda. . . . 157

11 Schleberoda . . . . 158

12 Waldgebiet Neue Göhle 159

13 Naturschutzgebiet

Müchelholz . . . . . 160

14 St. Micheln . . . . . . 161

15 Geiselquelle. . . . . . 162

16 Freyburger Unstruttal und

Zscheiplitzer Talung . . 165

17 Balgstädt . . . . . . 167

18 Zscheiplitz . . . . . . 169
C 19 Schweigenberge . . . 171

20 Freyburg. . . . . . . 173

21 Nißmitz . . . . . . . 179

22 Freyburger Plateau, Zeuchfelder Talung und

Pödelister Talung . . . 180

23 Zeuchfeld . . . . . . 181

24 Naturschutzgebiet Neue

Göhle . . . . . . . 183

25 Pödelist . . . . . . . 184

26 Hasselbachtal und

Kösener Plateau . . . 185 